\title{
Numerical Calculation of Certain Definite Integrals by Poisson's Summation Formula
}

It is generally agreed that of all quadrature formulae, the trapezoidal rule, while being the simplest, is also the least accurate. There is, however, a rather general class of integrals for which the trapezoidal rule can be shown to be a highly accurate means of obtaining numerical values. Specifically, if the integrand is a periodic, even function with all derivatives continuous, then the integral over a period is given by the trapezoidal rule with high precision. Similarly, integrals of the form $\int_{0}^{\infty} e^{-x^{2}} f(x) d x$, where $f(x)$ is an even, entire function of $x$ can be evaluated by this method.

The basis for the above statements is the Poisson summation formula [6] which reads

$$
\begin{gathered}
\int_{0}^{a} f(t) d t=h\left\{\frac{1}{2}[f(0)+f(a)]+\sum_{k=1}^{n-1} f(k h)\right\}-2 \sum_{k=1}^{\infty} g\left(\frac{2 k \pi}{h}\right), \\
h=a / n, \quad g(x)=\int_{0}^{a} f(t) \cos (x t) d t .
\end{gathered}
$$

This formula may be interpreted as the trapezoidal rule with a correction. The magnitude of the correction will depend on the manner in which the Fourier coefficients, $g(x)$, decrease with increasing $x$. Now if $f(t)$ is periodic, of period $a$ with all derivatives continuous, then it is known from principles of Fourier series that the coefficients in the series for $f(t)$ tend to zero at least as fast as $e^{-x}$ for $x$ sufficiently large. If this is the case, the first term in the correction to the trapezoidal rule will be of the order of $e^{-\frac{2 \pi}{h}}$, which is small even when " $h$ " is comparatively large. Thus, if it is possible to estimate in advance the order of magnitude of $g(x)$ for large values of $x$, an interval of integration may be determined to give any desired degree of accuracy.

If, in Poisson's formula, $a$ and $n$ are made to tend to infinity in such a way that $h$ remains fixed, there is obtained the result

$$
\begin{aligned}
\int_{0}^{\infty} f(x) d x & =h\left\{\frac{1}{2} f(0)+\sum_{k=1}^{\infty} f(k h)\right\}-2 \sum_{k=1}^{\infty} g\left(\frac{2 k \pi}{h}\right) \\
g(x) & =\int_{0}^{\infty} f(t) \cos (x t) d t .
\end{aligned}
$$

Thus, if $f(x)$ is an even function such that its Fourier transform $g(x)$ is negligibly small for large $x$, the trapezoidal rule may again be used with an arbitrarily small error.

We consider now some functions to which the above may be applied. 
(1) The Bessel Function $J_{p}(z)$, of Integral Order. This function may be defined by the definite integral as follows:

$$
J_{p}(z)=\frac{(i)^{n}}{\pi} \int_{0}^{\pi} e^{i z \cos t} \cos (p t) d t
$$

where " $p$ " is a positive integer.

Thus

$$
\begin{aligned}
f(t) & =e^{i z \cos t} \cos (p t) \\
g(x) & =\int_{0}^{\pi} e^{i z \cos t} \cos (p t) \cos (x t) d t \\
& =\frac{\pi}{2}\left[i^{(x+p)} J_{x+p}(z)+i^{(x-p)} J_{x-p}(z)\right],
\end{aligned}
$$

$(x$ an integer).
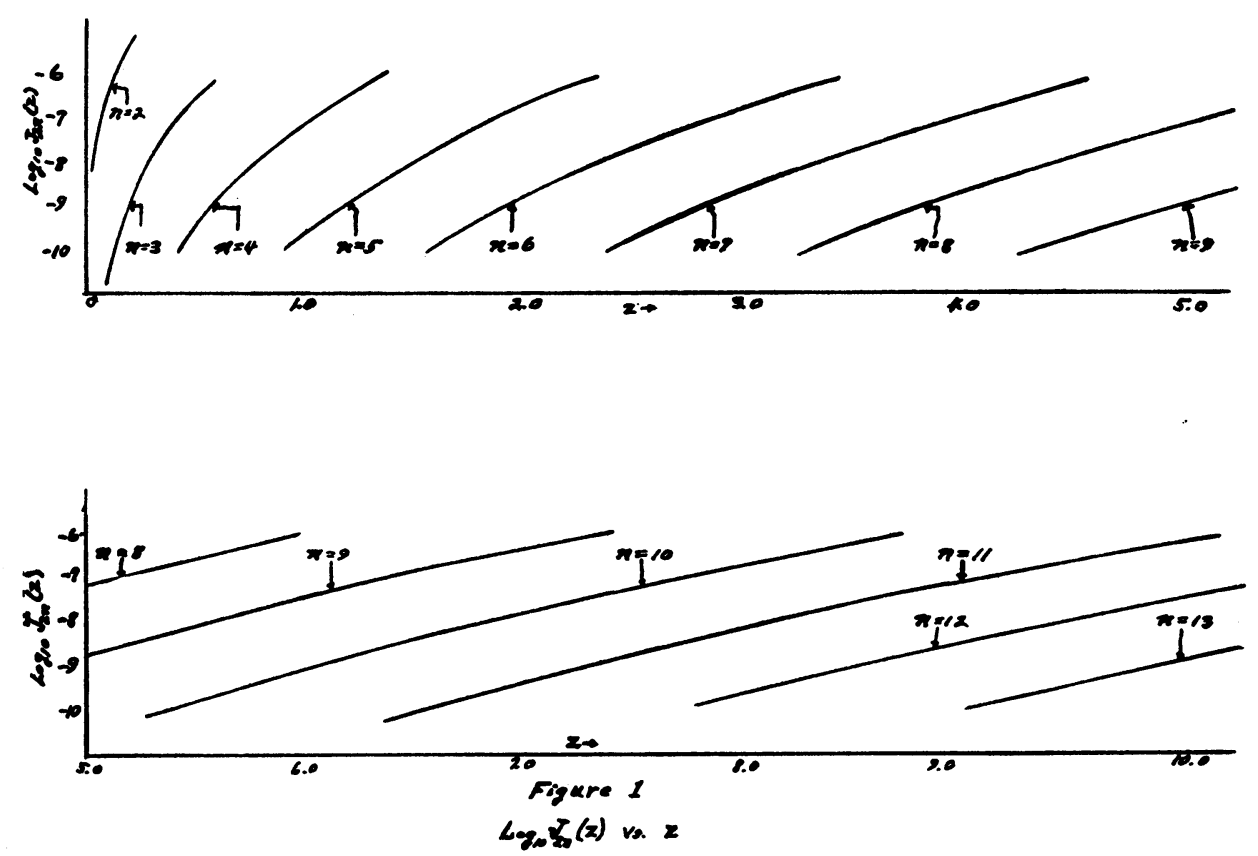

To obtain an error estimate from (4), it is noted that if " $x$ " is chosen sufficiently large, so that $J_{x+p}(z) \ll J_{x-p}(z)$, then the order of magnitude of $g(x)$ is essentially that of $J_{x-p}(z)$, and this in turn may be made arbitrarily small by the proper choice of " $x$," assuming " $p$ " and " $z$ " fixed. Therefore, the order of magnitude of the error, for which only the first term in the infinite series in (2) need be considered, is given by $J_{x-p}(z)$, with $x=2 \pi / h$. But by definition, $h=\pi / n$, and so the error estimate in terms of the number of subdivisions, $n$, of the interval of integration is given by $J_{2 n-p}(z)$.

The value of " $h$ " thus determined permits $J_{p}(z)$ to be approximated by a finite sum of sines and cosines for any value of $z$ and $p$, and to any accuracy which may be required. We thus obtain from the Poisson summation formula a simple 
analytic approximation to the Bessel function of the first kind of integral order, involving only elementary functions. Such a representation provides a means of generating these functions on an automatic digital computer using only subroutines for the trigonometric functions. In figure 1 , the value of $\log _{10} J_{2 n}(z)$ is plotted as a function of $z$ for those values of $n$ for which $J_{2 n}(z)$ lies between $10^{-6}$ and $10^{-10}$. The use of this plot is as follows: Assume that it is desired to approximate $J_{0}(z)$ in the range $0 \leq z \leq 2$, with a prescribed error of the order of $10^{-8}$. From the figure we see that when $2 n=12$ (whence $h=\pi / 6$ ) the error is of the desired order of magnitude. Therefore we find that, for the range $0 \leq z \leq 2$,

$$
\begin{aligned}
\pi J_{0}(z) \simeq \frac{\pi}{6}\left[\frac{1}{2} e^{i z}+e^{i z \cos \pi / 6}+e^{i z \cos \pi / 3}+e^{i z \cos \pi / 2}\right. & \\
& \left.+\frac{1}{2} e^{-i z}+e^{i z \cos 5 \pi / 6}+e^{i z \cos 2 \pi / 3}\right],
\end{aligned}
$$

or

$$
J_{0}(z) \simeq \frac{1}{6}\left[\cos (z)+2 \cos \left(\frac{\sqrt{3}}{2} z\right)+2 \cos \left(\frac{1}{2} z\right)+1\right],
$$

correct to at least eight decimal places. To obtain ten decimal place accuracy we need only increase the number of subdivisions from 6 to 8 . For $z=1$, formula (5) gives

$$
J_{0}(1) \simeq .7651976867
$$

which is correct to nine places with an error of one unit in the tenth. The values obtained by applying respectively Simpson's rule and Weddle's rule, with the same interval, are

$$
\begin{aligned}
& \left.J_{0}(1)\right]_{2}=.76521 \\
& \left.J_{0}(1)\right]_{w}=.76572
\end{aligned}
$$

With $h=\pi / 15, J_{0}(z)$ is given correctly to ten places for all values of $z$ up to 11 by

$$
\begin{aligned}
J_{0}(z) \sim \frac{1}{15}\{\cos (z) & +2 \cos \left[z \cos \frac{\pi}{15}\right]+2 \cos \left[z \cos \frac{2 \pi}{15}\right] \\
& +2 \cos \left[z \cos \frac{\pi}{5}\right]+2 \cos \left[z \cos \frac{4 \pi}{15}\right] \\
& \left.+2 \cos \left[z \cos \frac{\pi}{3}\right]+2 \cos \left[z \cos \frac{2 \pi}{5}\right]+2 \cos \left[z \cos \frac{7 \pi}{15}\right]\right\}
\end{aligned}
$$

(2) The Modified Bessel Function $I_{p}(z)$. This function can be computed in a similar manner by replacing $z$ by $i z . I_{n}(z)$ does not tend to zero quite as fast as does $J_{n}(z)$ when $n \rightarrow \infty$ but the difference is not appreciable if $z$ is not large [12]. The approximation (5) gives

$$
I_{0}(z)=\frac{1}{6}\left[\cosh (z)+2 \cosh \left(\frac{\sqrt{3}}{2} z\right)+2 \cosh \left(\frac{1}{2} z\right)+1\right]
$$


to within $10^{-10}$ if $z<1.2$ and to within $10^{-7}$ if $z<2$. For $z=1$ we obtain

$$
\begin{array}{ll}
\text { From }(7): & I_{0}(1)=1.266065878 . \\
\text { From Simpson's Rule: } & I_{0}(1)=1.266051 . \\
\text { From Weddle's Rule: } & I_{0}(1)=1.26658 .
\end{array}
$$

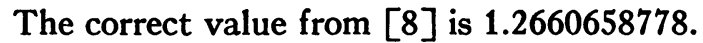

The approximations obtained in this way may also be used to evaluate integrals involving Bessel Functions. For example the functions considered by SCHWARTz [10] defined as

$$
\begin{aligned}
& J_{c}(\lambda, x)=\int_{0}^{x} J_{0}(\lambda t) \cos t d t, \\
& J_{s}(\lambda, x)=\int_{0}^{x} J_{0}(\lambda t) \sin t d t,
\end{aligned}
$$

may be evaluated in this way. If $x \leq 2$, the approximation (5) gives, after carrying out the integrations,

$$
\begin{aligned}
J_{c}(\lambda, x) \sim \frac{1}{6}\{\sin x & {\left[\frac{\cos (\lambda x)}{1-\lambda^{2}}+\frac{2 \cos \left(\frac{\sqrt{3}}{2} \lambda x\right)}{1-\frac{3}{4} \lambda^{2}}+\frac{2 \cos \left(\frac{1}{2} \lambda x\right)}{1-\frac{1}{4} \lambda^{2}}\right] } \\
& \left.-\lambda \cos x\left[\frac{\sin (\lambda x)}{1-\lambda^{2}}+\frac{\sqrt{3} \sin \left(\frac{\sqrt{3}}{2} \lambda x\right)}{1-\frac{3}{4} \lambda^{2}}+\frac{\sin \left(\frac{1}{2} \lambda x\right)}{1-\frac{1}{4} \lambda^{2}}\right]\right\} .
\end{aligned}
$$

A similar approximation may be obtained for $J_{s}(\lambda, x)$.

(3) The Modified Functions of the Second Kind, $K_{n}(z)$. These are defined by

$$
K_{n}(z)=\int_{0}^{\infty} e^{-z \cosh x} \cosh (n x) d x
$$

Hence

$$
\begin{aligned}
g(x) & =\int_{0}^{\infty} e^{-z \cosh t} \cosh (n t) \cos (x t) d t \\
& =\frac{1}{2}\left[K_{n+i x}(z)+K_{n+i x}(z)\right] .
\end{aligned}
$$

Now

$$
\frac{2}{\pi} K_{p}(z)=\frac{I_{p}(z)-I_{p}(z)}{\sin (p \pi)}
$$

Thus, using the approximation for $|p| \gg z$ :

$$
I_{p}(z) \sim \frac{(z / 2)^{p}}{\Gamma(p+1)},
$$


(12) $\frac{2}{\pi}\left[K_{n+i x}(z)+K_{-n+i x}(z)\right]$

$$
\begin{aligned}
& \sim \frac{(-)^{n}}{i \sinh (x \pi)}\left\{\left(\frac{z}{2}\right)^{-n}\right. {\left[\frac{e^{-i x \ln (z / 2)}}{\Gamma(1-n-i x)}-\frac{e^{i x \ln (z / 2)}}{\Gamma(1-n+i x)}\right] } \\
&\left.+\left(\frac{z}{2}\right)^{n}\left[\frac{e^{-i x \ln (z / 2)}}{\Gamma(1+n-i x)}-\frac{e^{i x \ln (z / 2)}}{\Gamma(1+n+i x)}\right]\right\} .
\end{aligned}
$$

Hence if

$$
\begin{aligned}
& \Gamma(1-n \pm i x)=|\Gamma(1-n+i x)| e^{ \pm i \varphi_{1}} \\
& \Gamma(1+n \pm i x)=|\Gamma(1+n+i x)| e^{ \pm i \varphi_{2}}
\end{aligned}
$$

then,

$$
\begin{aligned}
\frac{1}{\pi} \mid K_{n+i x}(z)+ & K_{-n+i x}(z) \mid \\
& \sim \frac{1}{\sinh (x \pi)}\left\{\left(\frac{z}{2}\right)^{-n} \frac{\sin \left(\varphi_{1}-x \ln \left(\frac{z}{2}\right)\right)}{|\Gamma(1-n+i x)|}\right. \\
& \left.+\left(\frac{z}{2}\right)^{n} \frac{\sin \left(\varphi_{2}-x \ln \left(\frac{z}{2}\right)\right)}{|\Gamma(1+n+i x)|}\right\} \\
& \leq \frac{1}{\sinh (x \pi)}\left\{\frac{\left(\frac{z}{2}\right)^{-n}}{|\Gamma(1-n+i x)|}+\frac{\left(\frac{z}{2}\right)^{n}}{|\Gamma(1+n+i x)|}\right.
\end{aligned}
$$

But

$$
|\Gamma(1+n+i x)|^{2}=\left[n^{2}+x^{2}\right]\left[(n-1)^{2}+x^{2}\right] \cdots\left[1+x^{2}\right]\left[x^{2}\right]|\Gamma(i x)|^{2}
$$

$$
\begin{aligned}
|\Gamma(1-n+i x)|^{2} & =\frac{|\Gamma(i x)|^{2}}{\left[(n-1)^{2}+x^{2}\right] \cdots\left[1+x^{2}\right]}, & & n>1 \\
& =|\Gamma(i x)|^{2}, & & n=1 \\
& =x^{2}|\Gamma(i x)|^{2} . & & n=0 .
\end{aligned}
$$

Therefore, since $|\Gamma(i x)|^{2}=\frac{\pi}{x \sinh (\pi x)}$,

$$
\begin{aligned}
\left|K_{n+i x}(z)+K_{-n+i x}(z)\right| \sim \frac{\sqrt{\pi}}{\sqrt{x \sinh (\pi x)}}\left\{\frac{\left(\frac{z}{2}\right)^{n}}{\sqrt{\left[1+x^{2}\right] \cdots\left[n^{2}+x^{2}\right]}}\right. \\
\left.+\frac{\sqrt{x^{2}\left(1+x^{2}\right)\left(4+x^{2}\right) \cdots\left[(n-1)^{2}+x^{2}\right]}}{\left(\frac{z}{2}\right)^{n}}\right\}
\end{aligned}
$$


for $n>1$;

$$
\begin{aligned}
\left|K_{1+i x}(z)+K_{-1+i x}(z)\right| & \sim \frac{\sqrt{\pi x}}{\sqrt{\sinh (\pi x)}}\left\{\frac{\left(\frac{z}{2}\right)}{x \sqrt{1+x^{2}}}+\frac{2}{z}\right\} \\
\left|K_{i x}(z)\right| & \sim \frac{\sqrt{\pi}}{\sqrt{x \sinh (\pi x)}} .
\end{aligned}
$$

The last result agrees with a more accurate expression for $K_{i x}(z)$ when $x \gg z$ (cf. 4, Art. 7.13.2, formula 19). It may also be noted that, because of the assumption $x \gg z$, the first term in braces of (15) is negligible in comparison to the second. If in addition, $x \gg n$, then

$$
\left|K_{n+i x}(z)+K_{-n+i x}(z)\right| \sim \sqrt{\frac{\pi}{x \sinh (\pi x)}}\left(\frac{2 x}{z}\right)^{n} .
$$

From either (15) or (16), it can be seen that for $n$ and $z$ fixed, and $x$ sufficiently large, an error of negligible magnitude can be obtained. For $n=0$, the error estimate is independent of $z$ provided the condition $x \gg z$ is satisfied. For example, with $h=.5$, we find that $x=4 \pi$, and an estimated error of

$$
\sqrt{\frac{1}{4 \sinh \left(4 \pi^{2}\right)}}=2.1 \times 10^{-9} \text {. }
$$

In some problems it is more convenient to work with $e^{z} K_{0}(z)$ rather than with $K_{0}(z)$ itself. Evidently, to obtain this quantity to the same accuracy will require a smaller interval if $z$ is large. Thus $h=.5$ and $z=.2$ gives

$$
e^{z} K_{0}(z) \sim 2.1407573184
$$

as compared to the tabulated value from [8] of 2.14075 73163. If $z=10$, an interval of $h=.25$ will give $e^{z} K_{0}(z)$ with a comparable error. By actual calculation the value .391631933 is obtained which agrees with Watson's value [11] to as many places as are tabulated there.

(4) The Error Function. This function is defined by

$$
H(z)=\frac{2}{\sqrt{\pi}} \int_{0}^{z} e^{-t^{2}} d t .
$$

It can also be shown $[6]$, that,

$$
H(z)=1-\frac{2 z}{\pi} e^{-z^{2}} \int_{0}^{\infty} \frac{e^{-t^{2}} d t}{z^{2}+t^{2}}
$$

which is in a form suitable for evaluation by the present method. Taking $f(t)=\frac{e^{-t^{2}}}{z^{2}+t^{2}}$, then $[4]$

$$
g(x)=\frac{\sqrt{\pi}}{2 z} e^{z^{2}}\left[e^{-x z} \int_{z-\frac{1}{2} x}^{\infty} e^{-t^{2}} d t+e^{x z} \int_{z+\frac{1}{2} x}^{\infty} e^{-t^{2}} d t\right]
$$


For large values of $x$ the first integral is nearly equal to $\int_{-\infty}^{\infty} e^{-t^{2}} d t=\sqrt{\pi}$ while the second is of the order of $\frac{e^{-\left(z+\frac{1}{3} x\right)^{2}}}{2 z+x}$. But since

therefore

$$
\left(z^{2}+\frac{1}{4} x^{2}\right) \geqslant z x,
$$

$$
\begin{aligned}
e^{-z x} & \geqslant e^{z x} e^{-\left(z+\frac{1}{2} x\right)^{2}} \\
& >e^{z x} \frac{e^{-\left(z+\frac{1}{z} x\right)^{2}}}{2 z+x} .
\end{aligned}
$$

Thus the error in computing the values of $\int_{0}^{\infty} \frac{e^{-t^{2}} d t}{t^{2}+z^{2}}$ with an interval " $h$ " is of the order of

$$
\frac{\pi}{2 z} e^{z^{2}-\frac{2 \pi z}{h}}
$$

while the error for $H(z)$ itself is approximately

$$
e^{-\frac{2 \pi z}{h}}
$$

Hence, to compute $H(z)$ with a prescribed error of (say) $\epsilon$, the value of $h$ must be so chosen that

$$
e^{-\frac{2 \pi z}{h}}<\epsilon
$$

For example, if $z^{2}=2$, we find that with $h=.5$, the error to be expected is $e^{-17.8}$ of $5 \times 10^{-8}$, while with $h=.25$, the anticipated error will be $2.5 \times 10^{-15}$. Actual calculations give:

$$
\text { with } \begin{aligned}
h & =.5 \\
H(\sqrt{2}) & =.04550030: \\
\text { with } \quad h & =.25 \\
H(\sqrt{2}) & =.0455002639 .
\end{aligned}
$$

The second value agrees exactly with the one tabulated in [9].

In the present paper, we have considered only a few of the many functions for which the trapezoidal rule provides a simple and highly accurate means of obtaining numerical values.

Wright Air Development Center

Henry E. Fettis

Wright-Patterson Air Force Base, Ohio

1 E. C. Titchmarsh, Theory of Functions, Oxford Univ. Press, London, 1939.

2 Smithsonian Mathematical Formulae and Tables of Elliptic Functions, Smithsonian Institution, Washington, 1922.

${ }^{3}$ H. B. DwIGHT, Tables of Integrals and Other Mathematical Data, Macmillan, New York, 1947.

4 A. Erdélyi, W. Magnus, F. Oberhettinger, \& F. G. Tricomi, Tables of Integral Transforms, McGraw-Hill, New York, 1954.

5 A. Erdelyi, W. Magnus, F. Oberhettinger, \& F. G. Tricomi, Higher Transcendental Functions, vol. 2, McGraw-Hill, New York, 1954. 
${ }^{6}$ Wilhelm Magnus \& Fritz Oberhettinger, Formeln und Sätze für die Speziellen Funktionen der Mathematischen Physik, Springer, Berlin, 1948.

7 F. JaHnke \& F. EMDE, Tables of Functions, Dover, New York, 1945.

${ }^{8}$ NBSCL, Tables of Bessel Functions $Y_{0}(z)$ and $Y_{1}(z)$ for Complex Arguments, Columbia Press, New York, 1950.

9 NBS Applied Mathematics Series No. 23, Tables of the Normal Probability Function, U. S. Govt. Printing Office, Washington, 1953.

${ }^{10} \mathrm{~L}$. SchwaRz, Untersuchung einiger mit den Zylinderfunktionen nullter Ordnung verwandter Funktionen. Luftfahrtforschung, v. 20,1944, p. 341-372.

$11 \mathrm{G}$. N. Watson, A Treatise on the Theory of Bessel Functions, Macmillan, New York, 1948.

12 For those values of $z$ and $n$ which are of interest here, the difference between $\log _{10} J_{n}(z)$ and $\log _{10} I_{n}(z)$ is approximately equal to $z^{2} \log _{10} e / 2(n+1)$.

\section{Trapezoidal Methods of Approximating Solutions of Differential Equations}

Introduction. Seemingly by historical accident the points of view usually adopted for stepwise methods of numerical solution of differential equations largely emphasize the discrete values found rather than the functions pieced together by the method over various intervals of advance. The authors, collaborating as a professor and student team, have found that the straightforward selection of functions to approximate solution functions offers many advantages conceptually and makes it possible to see much-used methods in a new light. We present here a few of the simpler results.

The purpose of this paper is to show that, by considering the method called the trapezoidal method (cf. MILnE [1] p. 24) as a parabolic or quadratic function method, not only does one obtain a satisfying geometrical picture of an approximation curve in the usual case, but that two new trapezoidal or parabolic methods are suggested. Of the two new methods, one is based on a Gauss integration formula. Thus the new approach makes it possible to use the Gauss integration formulas. We believe that the two methods are new in their application although they are old in their use of polynomials. We have found in certain applications that both the new formulas have points of preference in some cases over the simple trapezoidal method.

Quadratic Functions and Trapezoids. Let $y^{\prime}=f(x, y)$ and let it be supposed that $\left(x_{0}, y_{0}\right)$ is either an initial point or that we are now to treat it as such and that we wish to establish a value approximating the solution for $x=x_{1}$. Then, using Milne's terminology, we write

$$
y_{1}-y_{0}=\frac{h}{2}\left(y_{1}^{\prime}+y_{0}^{\prime}\right), \quad h=x_{1}-x_{0} .
$$

From (1) it is clear that, if the solution were known, then (1) is a trapezoidal rule for establishing the integral from the integrand values at the ends of the interval. In case of a differential equation in which $f(x, y)$ involves $y$, however, we do not know $y_{1}$ and if we agree to use $y_{1}{ }^{\prime}=f\left(x_{1}, y_{1}\right)$ we do not know $y_{1}{ }^{\prime}$. Hence, (1) may be considered as an implicit equation for $y_{1}$ which, with adequate restrictions on $h$, yields directly an iteration procedure converging to a unique value $y_{1}$.

Now it is clear that in general $y_{1}$, as finally obtained, is not exactly the value 